\title{
miR-506 suppresses neuroblastoma metastasis by targeting ROCK1
}

\author{
DIANGUO LI ${ }^{1 *}$, YANHUA CAO ${ }^{2 *}$, JINLIANG LI $^{1}$, JIALONG XU ${ }^{1}$, QIAN LIU ${ }^{1}$ and XIAOGANG SUN ${ }^{1}$ \\ ${ }^{1}$ Department of Pediatric Surgery, Second Hospital of Shandong University, Jinan, Shandong 250033; \\ ${ }^{2}$ Department of Pediatrics, General Hospital of Jinan Command, Jinan, Shandong 250031, P.R. China
}

Received December 27, 2014; Accepted January 28, 2016

DOI: $10.3892 / \mathrm{ol} .2016 .5442$

\begin{abstract}
Neuroblastoma is a complex form of cancer with highly heterogeneous clinical behavior that arises during childhood from precursor cells of the sympathetic nervous system. In patients with neuroblastoma, mortality often occurs as a result of metastasis. The disease predominantly spreads to bone marrow, with a survival rate of $\sim 40 \%$. The current study demonstrates that microRNA (miR)-506 directly targets and downregulates Rho-associated, coiled-coil containing protein kinase 1 (ROCK1) in transforming growth factor (TGF)- $\beta$ non-canonical pathways. It may be concluded that ROCK1 contributes to the invasion and migration of neuroblastoma cells by directly downregulating miR-506; thus, leading to the upregulation of ROCK1, which promotes cell invasion and migration. The present results provide a novel understanding of how miR-506 directly regulates TGF- $\beta$ non-canonical signaling.
\end{abstract}

\section{Introduction}

Neuroblastoma is one of the most prevalent types of cancer that develops in young children, and is characterized by aggressive progression, maturation or spontaneous regression. At diagnosis, neuroblastoma usually presents with metastasis and a poor outcome $(1,2)$. A key stage of neuroblastoma metastasis is the invasion of the cells of the primary tumor, which have advanced invasive and migratory abilities, into the surrounding normal tissues (3). At present, a number of microRNAs (miRNAs or miRs), including miR-10b, miR29a/b, miR-335, miR-7 and miR-338-3p, have been identified as being associated with tumor progression in neuroblastoma (4). However, the precise mechanisms that underlie this process, particularly the crucial molecular pathways, remain to be elucidated.

Correspondence to: Dr Dianguo Li, Department of Pediatric Surgery, Second Hospital of Shandong University, 247 Beiyuan Road, Jinan, Shandong 250033, P.R. China

E-mail: dianguoli@126.com

*Contributed equally

Key words: miR-506, ROCK1, neuroblastoma, metastasis
Similar to other types of cancer, neuroblastoma development is a complex process with accumulation of epigenetic and genetic changes. The modified expression of miRNAs, including miR-148a, miR-21 and miR-200a, has been identified to modulate cell growth, migration, invasion and apoptosis in neuroblastoma $(5,6)$. Thus, additional investigation into the role of miRNAs is required in order to establish their function in the development of neuroblastoma $(7,8)$.

Previously, a miRNA assay identified the novel miRNA miR-506, which regulated neuroblastoma cell differentiation (9); however, the role of miR-506 in the development of the disease remains unknown. In the current study, the biological function of miR-506 in neuroblastoma was investigated. The results demonstrated that Rho-associated, coiled-coil containing protein kinase 1 (ROCK1) is a target of miR-506, and the expression of ROCK1 is positively associated with metastasis in patients with neuroblastoma. In addition, in vitro experiments demonstrated that ROCK1 is implicated in neuroblastoma cell invasion and motility. These results suggest that miR-506 functions as a tumor suppressor, inhibiting neuroblastoma metastasis by directly targeting ROCK1.

\section{Materials and methods}

Clinical specimens. Primary and normal biopsy specimens from children with neuroblastoma were obtained from the Second Hospital of Shandong University (Jinan, China). The identification of the tumor and normal tissues was histologically confirmed by hematoxylin and eosin staining. Informed consent was obtained from each patient, and the research protocols were approved by the Ethics Committee of the Second Hospital of Shandong University.

Cell culture and antibodies. IMR-32, N2A, SK-N-SH and SH-SY5Y cells were originally obtained from the American Type Culture Collection (Manassas, VA, USA), and were cultured in Dulbecco's modified Eagle's medium (Gibco; Thermo Fisher Scientific, Inc., Waltham, MA, USA) supplemented with $10 \%$ fetal bovine serum (Gibco; Thermo Fisher Scientific, Inc.), $100 \mathrm{U} / \mathrm{ml}$ penicillin and $100 \mu \mathrm{g} / \mathrm{ml}$ streptomycin (Sigma-Aldrich, St. Louis, MO, USA). The cells were cultured at $37^{\circ} \mathrm{C}$ in a $5 \% \mathrm{CO}_{2}$ humidified atmosphere. ROCK1 primary monoclonal rabbit antibody (1:1,000 dilution) was purchased from Cell Signaling Technology, Inc. (Danvers, 
MA, USA; catalog no., 4035). Glyceraldehyde 3-phosphate dehydrogenase (GAPDH) was purchased from Santa Cruz Biotechnology, Inc. (Dallas, TX, USA).

Transfection. miRNA mimics and miRNA antagomiRs were designed and synthesized by Guangzhou RiboBio Co., Ltd. (Guangzhou, China). The miRNA antagomiRs were composed of nucleotides with a 2'-O-methyl modification. Cells were transiently transfected with miRNA mimics and miRNA antagomiRs using Invitrogen Lipofectamine ${ }^{\circledR} 2000$ (Thermo Fisher Scientific,Inc.), according to the manufacturer's protocol.

$R N A$ isolation and reverse transcription-quantitative polymerase chain reaction (RT- $q P C R)$. Following the manufacturer's protocol, total RNA was isolated from the cells using Invitrogen TRIzol ${ }^{\circledR}$ Reagent (Thermo Fisher Scientific, Inc.). A total of $2 \mu \mathrm{g}$ RNA was treated with DNase (Promega Corporation, Madison, WI, USA) to remove contaminating DNA prior to RT of the RNA to cDNA, which was completed using the One Step SYBR ${ }^{\circledR}$ PrimeScript ${ }^{\mathrm{TM}}$ RT-PCR kit (Takara Bio, Inc., Otsu, Japan). RT was performed with specific primers, with the reaction mixtures incubated at $16^{\circ} \mathrm{C}$ for $30 \mathrm{~min}, 42^{\circ} \mathrm{C}$ for $30 \mathrm{~min}$ and $85^{\circ} \mathrm{C}$ for $5 \mathrm{~min}$. To measure messenger (m)RNA expression, RT-qPCR was performed using the ABI PRISM 7900HT Sequence Detection system (Applied Biosystems; Thermo Fisher Scientific, Inc.). Primers were purchased from Invitrogen (Thermo Fisher Scientific, Inc.). Primer sequences were as follows: Forward, 5'-TAAGGCACCCTTCTGAGT AGA-3' and reverse, 5'-GCGAGCACAGAATTAATACGA C-3' for miR-506; forward, 5'-AGAGCCTGTGGTGTCCG-3' and reverse 5'-CATCTTCAAAGCACTTCCCT-3' for U6; forward, 5'-AACCATGTGACTGAGTGCCC-3' and reverse, 5'-TCAGTGTGTTGTGCCAAAGC-3' for ROCK1; and forward 5'-AATCCCATCACCATCTTCCA-3' and reverse, 5'-TGGACTCCACGACGTACTCA-3' for GAPDH. PCR cycling conditions were as follows: An initial denaturation step at $95^{\circ} \mathrm{C}$ for $10 \mathrm{~min}$, followed by 40 cycles of denaturing at $95^{\circ} \mathrm{C}$ for $10 \mathrm{sec}$, annealing and synthesis at $60^{\circ} \mathrm{C}$ for $60 \mathrm{sec}$. The relative expression levels were calculated by comparing $\mathrm{Cq}$ values of the samples with those of the reference $\left(2^{-\Delta \Delta C q}\right)$, and all data were normalized to the internal control GAPDH (10).

Methyl thiazolyl tetrazolium (MTT) assay. An MTT assay was used to detect the growth of cells, and the growth curve was delineated. Logarithmic phase cells were collected, and the concentration of the cell suspension was adjusted to 5,000 cells per well. The wells at the edge of the plate were filled with aseptic phosphate-buffered saline. The cells were incubated at $37^{\circ} \mathrm{C}$ with $5 \% \mathrm{CO}_{2}$ and were cultured once they had covered the bottom of the well in a flat-bottom 96-well plate (Corning Incorporated, Corning, NY, USA). A total of $20 \mu 1$ MTT solution was added to each well $(5 \mathrm{mg} / \mathrm{ml} ; 0.5 \%$ MTT; Sigma-Aldrich), and the cells were cultured for $4 \mathrm{~h}$ at $37^{\circ} \mathrm{C}$. Following incubation, the supernatant was discarded and $150 \mu 1$ dimethyl sulfoxide (Sigma-Aldrich) was added to each well, and the culture plate was agitated at a low speed for 10 min until the crystal had dissolved completely. An enzyme-linked immunosorbent assay reader (MR-201 ELISA Microplate Reader) was used to measure the absorbance at $570 \mathrm{~nm}$.
Western blot analysis. Western blots were performed as previously described (11). Cells were lysed in radioimmunoprecipitation assay buffer (Sigma-Aldrich), containing 1X protease inhibitor cocktail (Sigma-Aldrich), and protein concentrations were determined using the Quick Start ${ }^{\mathrm{TM}}$ Bradford protein assay (Bio-Rad Laboratories, Inc., Hercules, CA, USA). Proteins were separated by $12.5 \%$ sodium dodecyl sulfate polyacrylamide gel electrophoresis and transferred to membranes (EMD Millipore, Billerica, MA, USA) at $55 \mathrm{~V}$ for $4 \mathrm{~h}$ at $4^{\circ} \mathrm{C}$. Subsequent to blocking in 5\% non-fat dry milk in Tris-buffered saline (TBS; Sigma-Aldrich), the membranes were incubated with primary antibodies in a 1:1,000 dilution in TBS overnight at $4^{\circ} \mathrm{C}$. The membranes were then washed 3 times with TBS-Tween 20 (Sigma-Aldrich), and incubated with secondary antibodies conjugated with horseradish peroxidase in a 1:5,000 dilution in TBS for $1 \mathrm{~h}$ at room temperature. The membranes were then washed 3 times in TBS-Tween 20 at room temperature. The protein bands were visualized on $\mathrm{X}$-ray film using an enhanced chemiluminescence detection system (Bio-Rad Laboratories, Inc., Hercules, CA, USA).

Migration assay. For the Transwell migration assay, $1 \times 10^{5}$ cells were plated into the top chamber of the non-coated membrane (24-well insert; pore size, $8 \mu \mathrm{m}$; Corning Incorporated) and allowed to migrate towards the serum-containing medium in the lower chamber. Cells were fixed following $24 \mathrm{~h}$ of incubation with methanol and stained with $0.1 \%$ crystal violet ( $2 \mathrm{mg} / \mathrm{ml}$; Sigma-Aldrich). A total of 3 random fields per well were selected, and using a light microscope (x40 magnification; BX51; Olympus Corporation, Tokyo, Japan), the number of cells invading through the membrane was counted.

Invasion assay. For the invasion assay, $1 \times 10^{5}$ neuroblastoma cells were plated in the upper-chamber with a Matrigel-coated membrane (24-well insert; pore size, $8 \mu \mathrm{m}$; BD Biosciences, San Jose, CA, USA). Each well was freshly coated with Matrigel (60 $\mu \mathrm{g}$; BD Biosciences) prior to the invasion assay. The cells were plated in medium without serum or growth factors, whilst medium supplemented with serum was used as a chemoattractant in the lower chamber. The cells were incubated for $48 \mathrm{~h}$ and cells that did invade through the pores were removed by a cotton swab. Cells on the lower surface of the membrane were fixed with methanol and stained with crystal violet. A total of 3 random fields were selected, and using a light microscope (x40 magnification; BX51), the number of cells invading through the membrane was counted.

Statistical analysis. All experiments were performed in triplicate. The data are expressed as the mean \pm standard error of the mean and were analyzed using Student's $t$-test. $\mathrm{P}<0.05$ was considered to indicate a statistically significant difference. Statistical analyses were performed using GraphPad Prism 5.01 software (GraphPad Software Inc., La Jolla, CA, USA).

\section{Results}

miR-506 expression is decreased in neuroblastoma tissues and cell lines. The role of miR-506 in neuroblastoma remains unknown. In order to investigate the role of miR-506, miR-506 
A

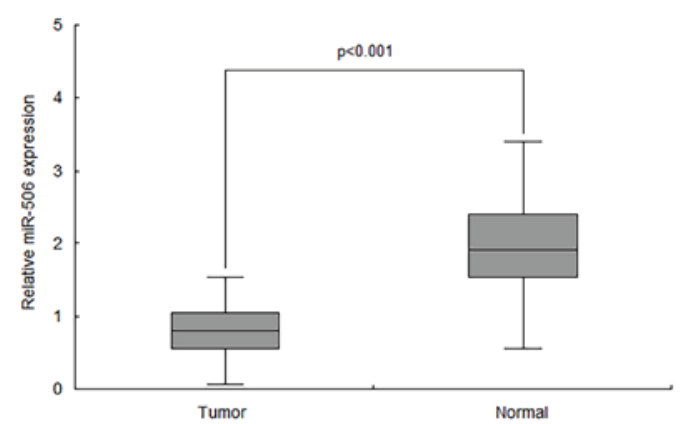



C

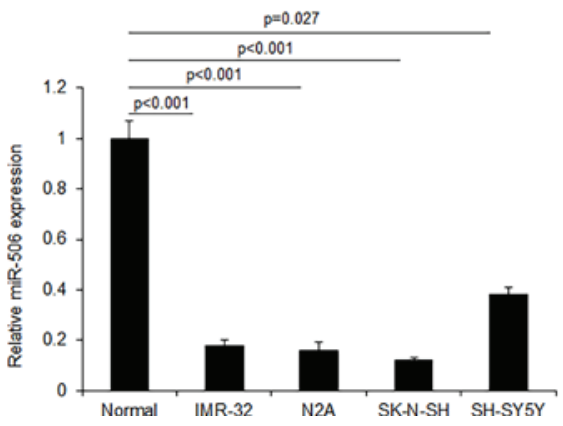

Figure 1. miR-506 expression decreases in neuroblastoma tissues and cell lines. miR-506 expression was examined by RT-qPCR. (A) miR-506 expression was observed to be lower in tumor tissues when compared with adjacent normal tissues from 48 patients. (B) miR-506 expression was lower in metastatic tissues when compared with non-metastatic tissues. (C) Analysis of miR-506 expression in neuroblastoma and normal cells. The data are expressed as the mean \pm standard deviation and were collected from three independent experiments. miR, microRNA; RT-qPCR, reverse transcription-quantitative polymerase chain reaction.

A

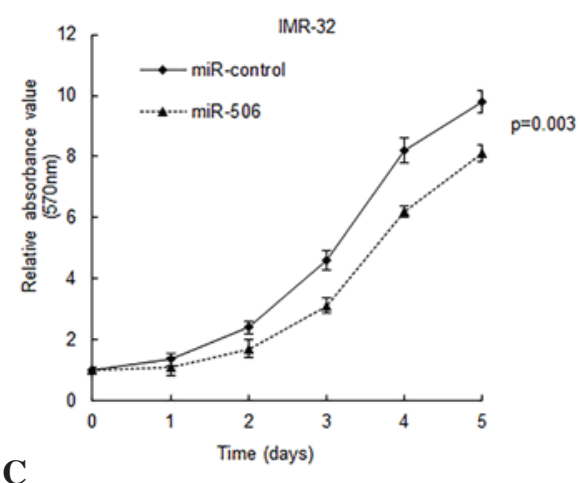

C



B

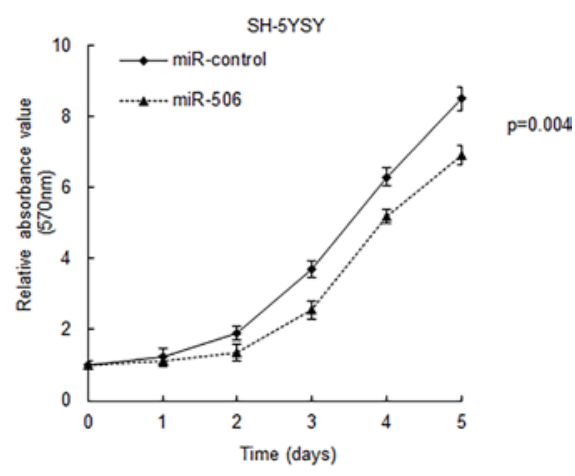

D

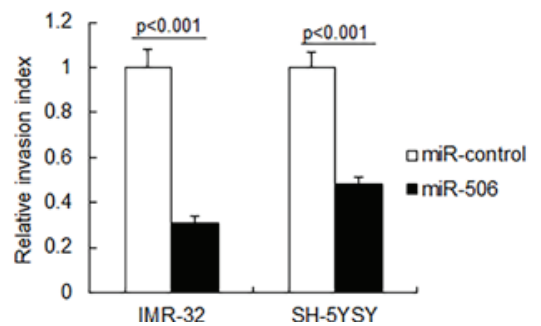

Figure 2. miR-506 inhibits metastasis in neuroblastoma cells. The effects of miR-506 on (A) IMR-32 and (B) SH-5YSY cell proliferation observed by methyl thiazolyl tetrazolium assay. (C) miR-506 inhibited the migratory ability of IMR-32 and SH-SY5Y cells, with suppressed cells passing through the membrane (D) miR-506 inhibited the invasive ability of IMR-32 and SH-SY5Y cells, with suppressed cells passing through the membrane. The chambers were coated with Matrigel, which functioned as the extracellular cell matrix. miR, microRNA.

expression was detected in neuroblastoma tissues using RT-qPCR. The results demonstrated that miR-506 expression decreased in $\sim 2 / 3$ of neuroblastoma tissues, compared with the normal tissues, and increased in $\sim 1 / 3$ of tissues (Fig. 1A). It was also observed that the loss of miR-506 expression was associated with metastasis in neuroblastoma tissue (Fig. 1B). 
A

B

Position 930-937 of ROCK1 3' UTR 5 '...UGCAUUGUCCUUUUAGUGCCUUA...

$|1| 1|1|$

Has-miR-506

3' AgaugagucuuccCACGgaAu

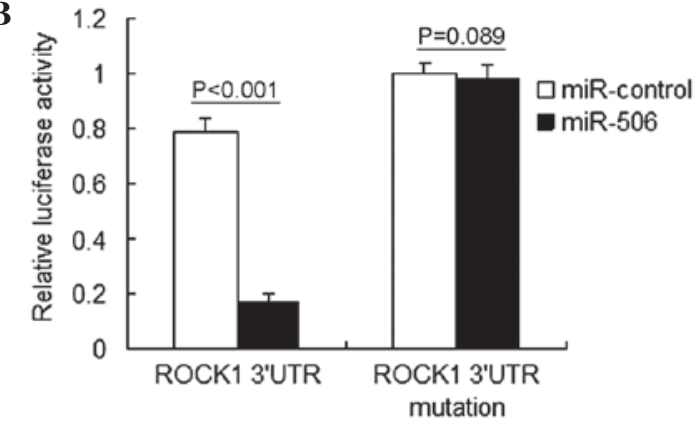

D


Figure 3. ROCK1 is a target gene of miR-506 in neuroblastoma cells. (A) According to bioinformatics analysis, the 3'-UTR of the ROCK1 gene contains binding sites for miR-506. (B) miR-506 suppressed the expression of a luciferase reporter gene harboring the 3'-UTR of ROCK1 in IMR-32 cells. The pGL4 plasmid was modified by adding either the human 3'-UTR or the 3'-UTR with mutations in regions complementary to miR-506 seed regions behind the firefly luciferase gene. IMR-32 cells were transiently co-transfected with a negative control (mock) or miR-506 together with the indicated luciferase constructs, and luciferase activity was analyzed $48 \mathrm{~h}$ later. Data are presented as relative firefly luciferase activity normalized to Renilla luciferase activity from the same construct. (C) miR-506 restoration downregulated ROCK1 in the neuroblastoma cells. The cells were transfected with miR-506 or miR control for $48 \mathrm{~h}$, and were then collected for reverse transcription-quantitative polymerase chain reaction. (D) miR-506 restoration downregulated ROCK1 expression in the neuroblastoma IMR-32 and SH-SY5Y cell lines. The cells were transfected with miR-506 or miR-control for $48 \mathrm{~h}$, and were then collected for western blot analysis. The data are presented as the mean \pm standard deviation and the data were collected from three independent experiments. ROCK1, Rho-associated, coiled-coil containing protein kinase 1; 3'-UTR, 3'-untranslated region; miR, microRNA; GAPDH, glyceraldehyde 3-phosphate dehydrogenase.

A

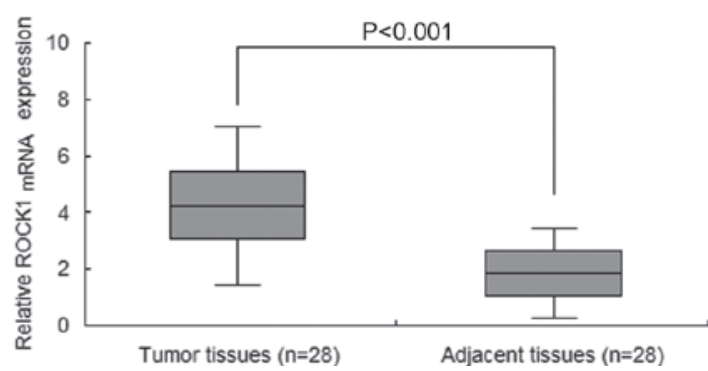

B

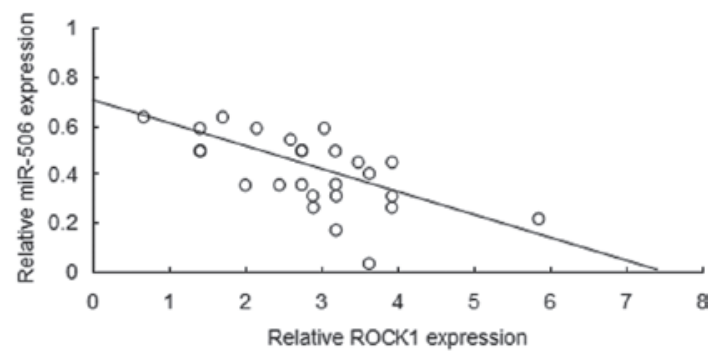

Figure 4. miR-506 expression was negatively associated with ROCK1 in neuroblastoma tissues. (A) ROCK1 protein was overexpressed in neuroblastoma tissues compared with the adjacent normal tissues. (B) miR-506 was negatively associated with ROCK1 mRNA in the neuroblastoma tissue. The data are presented as the mean \pm standard deviation and the data were collected from three independent experiments. ROCK1, Rho-associated, coiled-coil containing protein kinase 1 ; miR, microRNA.

Similar results were confirmed by RT-qPCR in the neuroblastoma cell lines (Fig. 1C). The downregulation of miR-506 in neuroblastoma tissue and cells indicates that miR-506 may function as a tumor suppressor gene.

miR-506 inhibits neuroblastoma proliferation and metastasis. To investigate the role of miR-506 in neuroblastoma cells, IMR-32 and SH-SY5Y cells were transfected with miR-506 and cell proliferation was observed by an MTT assay. As presented in Fig. 2A and B, miR-506 overexpression in the IMR-32 and SH-SY5Y cells resulted in a significant inhibition of cell proliferation. Metastasis is an important characteristic of neuroblastoma, thus cell migration and invasion were analyzed by Transwell migration assay. It was demonstrated that miR-506 significantly inhibited cell migration and invasion in the IMR-32 and SH-5YSY cells ( $<<0.001$; Fig. $2 \mathrm{C}$ and D).

ROCK1 is a target gene of miR-506 in neuroblastoma. A single miRNA, including miR-506, may regulate the expression of numerous target genes. Using Targetscan (http://www.targetscan.org) and miRBase (www.mirbase.org), multiple genes were predicted to be potential target genes of 
miR-506. The target genes with higher scores were selected for RT-qPCR analysis, and ROCK1 was noted as one of the downregulated genes (Fig. 3A). The present study focused on ROCK1 in particular due to its important role in various types of cancer, including neuroblastoma. Luciferase activity assay identified ROCK1 as the target gene of miR-506 in IMR-32 cells $(\mathrm{P}<0.001$; Fig. 3B). RT-qPCR and western blot analysis were performed to analyze ROCK $1 \mathrm{mRNA}$ and protein levels in neuroblastoma cells. It was demonstrated that ROCK1 mRNA $(\mathrm{P}<0.001$; Fig. 3C) and protein (Fig. 3D) levels decreased in the IMR-32 cells treated with miR-506. These results verified that ROCK1 was a direct target gene of miR-506.

miR-506 expression is negatively associated with ROCK1 in neuroblastoma tissues. To increase the understanding of the association between miR-506a and ROCK1 in neuroblastoma, ROCK1 mRNA levels were analyzed by RT-qPCR. The results demonstrated that the ROCK1 mRNA is significantly overexpressed in neuroblastoma tissues when compared with normal tissues $(\mathrm{P}<0.001$; Fig. $4 \mathrm{~A})$. In addition, miR-506 was negatively associated with ROCK 1 in the neuroblastoma tissue (Fig. 4B).

\section{Discussion}

miRNAs perform important functions in the regulation of gene expression (12). The majority of miRNAs are involved in regulating the biological functions of cancer, including tumorigenesis, invasion and recurrence $(13,14)$. The deletion or overexpression of miRNAs may induce the initiation and development of human cancer, including neuroblastoma (14). Due to the multiple mechanisms that may lead to the resistance of neuroblastoma to therapy, targeting single elements of a signalling pathway may not be a sufficient treatment. Therefore, it is desirable to identify molecules, including miRNAs, that are able to regulate multiple cellular processes. This may minimise the risk of resistance to therapy and improve the overall clinical response to treatment. miRNAs are upstream regulators that are able to simultaneously target large numbers of protein-coding genes and multiple cancer signaling pathways. Emerging cumulative data reveal fucntional roles of miRNAs in the origin and progression of neuroblastoma (14). In the present study, it was demonstrated that miR-506 functions as a tumor suppressor in neuroblastoma by directly targeting ROCK1.

Previous studies have reported that the expression of miR-506 is reduced in numerous types of cancer, including epithelial ovarian cancer (15-18), hepatocellular carcinoma (19), cervical cancer (20), lung cancer (21), breast cancer (22) and colon cancer (23), and is involved in cancer progression, notably cell proliferation, metastasis and apoptosis. In the current study, it was observed that miR-506 was decreased in neuroblastoma tissues and cell lines when compared to normal tissues, supporting the notion that miR-506 functions as a potential tumor suppressor. Additionally, the results demonstrated that miR-506 inhibited neuroblastoma cell proliferation and metastasis. The previously reported target genes of miR-506 include cyclin-dependent kinase4/6-forkhead box protein M1 (18), yes-associated protein (19), GLI family zinc finger 3 (20), nuclear factor- $\kappa$ B p65 (21) and others. In the present study, it was verified that ROCK1 was a novel target gene for miR-506 in neuroblastoma.

The Rho-associated serine/threonine kinase family, of which ROCK1 is a member, operates as a key regulator of actin cytoskeleton dynamics and organization (24). ROCK1 has various different roles, including involvement in invasion, metastasis and migration during tumorigenesis (25). Several miRNAs target ROCK1 in numerous types of cancer, including miRNA-148a in nasopharyngeal carcinoma (26) and miRNA-340 in pediatric osteosarcoma (27). ROCK1 has been positively correlated with lymph node metastasis and the tumor-node-metastasis stage, and is implicated in miRNA-148a-induced inhibition of gastric cancer cell invasion and migration (28). The present study demonstrated that ROCK1 is a predicted target gene of miR-506 in neuroblastoma cells, and acts as an oncogene. Furthermore, it was observed that ROCK1 expression was negatively associated with miR-506 in neuroblastoma tissue.

In conclusion, the present study indicates that miR-506 functions as a tumor suppressor in neuroblastoma and negatively regulates cell proliferation and metastasis. Furthermore, the current study verified that ROCK 1 is direct target gene of miR-506 in neuroblastoma. The expression of miR-506 is downregulated in neuroblastoma tissues and cell lines and may have significant functions in neuroblastoma suppression by downregulation of ROCK 1 . ROCK1 may be a novel therapeutic target molecule for the treatment of neuroblastoma.

\section{References}

1. Park JR, Bagatell R, London WB, Maris JM, Cohn SL, Mattay KK and Hogarty M; COG Neuroblastoma Committee: Children's Oncology Group's 2013 blueprint for research: Neuroblastoma. Pediatr Blood Cancer 60: 985-993, 2013.

2. Irwin MS and Park JR: Neuroblastoma: Paradigm for precision medicine. Pediatr Clin North Am 62: 225-256, 2015.

3. Tong JL, Zhang CP, Nie F, Xu XT, Zhu MM, Xiao SD and Ran ZH: MicroRNA 506 regulates expression of PPAR alpha in hydroxycamptothecin-resistant human colon cancer cells. FEBS Lett 585: 3560-3568, 2011.

4. Shalaby T, Fiaschetti G, Baumgartner M and Grotzer MA: Significance and therapeutic value of miRNAs in embryonal neural tumors. Molecules 19: 5821-5862, 2014.

5. Zhi F, Wang R, Wang Q, Xue L, Deng D, Wang S and Yang Y: MicroRNAs in neuroblastoma: Small-sized players with a large impact. Neurochem Res 39: 613-623, 2014.

6. Mei H, Lin ZY and Tong QS: The roles of microRNAs in neuroblastoma. World J Pediatr 10: 10-16, 2014.

7. Domingo-Fernandez R, Watters K, Piskareva O, Stallings RL and Bray I: The role of genetic and epigenetic alterations in neuroblastoma disease pathogenesis. Pediatr Surg Int 29: 101-119, 2013.

8. Verissimo CS, Molenaar JJ, Fitzsimons CP and Vreugdenhil E: Neuroblastoma therapy: What is in the pipeline? Endocr Relat Cancer 18: R213-R231, 2011.

9. Stallings RL, Foley NH, Bray IM, Das S and Buckley PG: MicroRNA and DNA methylation alterations mediating retinoic acid induced neuroblastoma cell differentiation. Semin Cancer Biol 21: 283-290, 2011.

10. Livak and Schmittgen: Analysis of relative gene expression data using real-time quantitative PCR and the $2-\Delta \Delta \mathrm{Ct}$ method. Methods 25: 402-408, 2001.

11. Cheng J, Xie HY, Xu X, Wu J, Wei X, Su R, Zhang W, Lv Z, Zheng $S$ and Zhou L: NDRG1 as a biomarker for metastasis, recurrence and of poor prognosis in hepatocellular carcinoma. Cancer Lett 310: 35-45, 2011. 
12. AlmeidaMI,Reis RM and Calin GA: MicroRNAs and metastases - the neuroblastoma link. Cancer Biol Ther 9: 453-454, 2010.

13. Schulte JH, Horn S, Schlierf S, Schramm A, Heukamp LC, Christiansen H, Buettner R, Berwanger B and Eggert A: MicroRNAs in the pathogenesis of neuroblastoma. Cancer Lett 274: 10-15, 2009.

14. Stallings RL: MicroRNA involvement in the pathogenesis of neuroblastoma: Potential for microRNA mediated therapeutics. Curr Pharm Des 15: 456-462, 2009.

15. Sun Y, Hu L, Zheng H, Bagnoli M, Guo Y, Rupaimoole R, Rodriguez-Aguayo C, Lopez-Berestein G, Ji P, Chen K, et al: MiR-506 inhibits multiple targets in the epithelial-to-mesenchymal transition network and is associated with good prognosis in epithelial ovarian cancer. J Pathol 235: 25-36, 2015.

16. Koutsaki M, Spandidos DA and Zaravinos A: Epithelial-mesenchymal transition-associated miRNAs in ovarian carcinoma, with highlight on the miR-200 family: Prognostic value and prospective role in ovarian cancer therapeutics. Cancer Lett 351: 173-181, 2014.

17. Yang D, Sun Y, Hu L, Zheng H, Ji P, Pecot CV,Zhao Y, Reynolds S, Cheng H, Rupaimoole R, et al: Integrated analyses identify a master microRNA regulatory network for the mesenchymal subtype in serous ovarian cancer. Cancer Cell 23: 186-199, 2013.

18. Liu G, Sun Y, Ji P, Li X, Cogdell D, Yang D, Parker Kerrigan BC, Shmulevich I, Chen K, Sood AK, et al: MiR-506 suppresses proliferation and induces senescence by directly targeting the CDK4/6-FOXM1 axis in ovarian cancer.J Pathol 233: 308-318, 2014

19. Wang Y, Cui M, Sun BD, Liu FB, Zhang XD and Ye LH: MiR-506 suppresses proliferation of hepatoma cells through targeting YAP mRNA 3'UTR. Acta Pharmacol Sin 35: 1207-1214, 2014.

20. Wen SY,Lin Y, Yu YQ, Cao SJ,Zhang R, Yang XM, Li J,Zhang YL, Wang YH, Ma MZ, et al: miR-506 acts as a tumor suppressor by directly targeting the hedgehog pathway transcription factor Gli3 in human cervical cancer. Oncogene 34: 717-725, 2015.
21. Zhao Z, Ma X, Hsiao TH, Lin G, Kosti A, Yu X, Suresh U, Chen Y, Tomlinson GE, Pertsemlidis A and Du L: A high-content morphological screen identifies novel microRNAs that regulate neuroblastoma cell differentiation. Oncotarget 5: 2499-2512, 2014

22. Yin M, Ren X, Zhang X, Luo Y, Wang G, Huang K, Feng S, Bao X, Huang K, He X, et al: Selective killing of lung cancer cells by miRNA-506 molecule through inhibiting NF- $\mathrm{B}$ p 65 to evoke reactive oxygen species generation and p53 activation. Oncogene 34: 691-703, 2015.

23. Arora H, Qureshi R and Park WY: miR-506 regulates epithelial mesenchymal transition in breast cancer cell lines. PLoS One 8: e64273, 2013.

24. Nunes KP, Rigsby CS and Webb RC: RhoA/Rho-kinase and vascular diseases: What is the link? Cell Mol Life Sci 67: 3823-3836, 2010.

25. Schofield AV and Bernard O: Rho-associated coiled-coil kinase (ROCK) signaling and disease. Crit Rev Biochem Mol Biol 48: 301-316, 2013.

26. Li HP, Huang HY, Lai YR, Huang JX, Chang KP, Hsueh C and Chang YS: Silencing of miRNA-148a by hypermethylation activates the integrin-mediated signaling pathway in nasopharyngeal carcinoma. Oncotarget 5: 7610-7624, 2014.

27. Cai H, Lin L, Cai H, Tang $M$ and Wang Z: Combined microRNA-340 and ROCK1 mRNA profiling predicts tumor progression and prognosis in pediatric osteosarcoma. Int $\mathrm{J}$ Mol Sci 15: 560-573, 2014.

28. Shin JY, Kim YI, Cho SJ, Lee MK, Kook MC, Lee JH, Lee SS, Ashktorab H, Smoot DT, Ryu KW, et al: MicroRNA 135a suppresses lymph node metastasis through down-regulation of ROCK1 in early gastric cancer. PLoS One 9: e85205, 2014. 